1 School of Health and Biological Sciences, Universidade Positivo, UP, Curitiba, PR, Brazil.
Corresponding author: Denise Piotto Leonardi Rua Prof. Pedro Viriato Parigot de Souza, 5300

81280-330 Curitiba, Paraná, Brazil. Tel: $+55413317-3888$

E-mail: dpleonardi@gmail.com

Received: March 27, 2018

Accepted: November 27, 2018

\section{Long-term evaluation of crown and root discoloration induced by different endodontic sealers}

\author{
Beatriz Serrato Coelho', Paula Pontes Garcia', Flávia \\ Sens Fagundes Tomazinho', Leonardo Fernandes \\ da Cunha ${ }^{1}$, Denise Piotto Leonardi ${ }^{1}{ }^{*}$, Carla Castiglia \\ Gonzaga ${ }^{1}$, Marilisa Carneiro Leão Gabardo ${ }^{1}$, Flares \\ Baratto-Filho'
}

Aim: To evaluate crown and root discoloration promoted by different endodontic sealers after root canal filling. Methods: Eighty bovine incisors were prepared and filled with: Endofill, Sealer 26, AH Plus, and MTA Fillapex. Color was recorded using a spectrophotometer before endodontic treatment (TO) and at 24 hours (T1), seven days (T2), 30 days (T3), and 90 days (T4) after treatment. Analyses were performed on the middle and cervical regions of the crown, and on the cervical third of the root, immediately below the cementoenamel junction. The color alterations $(\Delta \mathrm{E})$ were calculated using Commission International de l'Eclairage (CIE) $L^{*} a^{*} b$ parameters, and data were analyzed by analysis of variance (ANOVA) and Tukey's test $(p<0.05)$. Results: All sealers induced color alterations. Sealer 26 resulted in the smallest changes in color (DE $=5.32)$. The other materials did not present statistical differences (AH Plus DE = 6.98; MTA Fillapex DE = 6.88; Endofill DE =6.41). Of the three regions analyzed, the largest discoloration was observed at the cervical third of the root (DE=10.67). In terms of time, the largest $\Delta E$ values ( $D E=7.72)$ were observed at $T 4$. Color changes at $\mathrm{T} 1$ ( $\mathrm{DE}=5.88), \mathrm{T} 2(\mathrm{DE}=6.10)$, and $\mathrm{T} 3(\mathrm{DE}=5.89)$ were statistically similar. Conclusions: All endodontic sealers promoted discoloration on the tooth crown and root.

Keywords: Endodontics. Root canal filling materials. Tooth crown. Tooth discoloration. 


\section{Introduction}

Tooth discoloration can occur due to penetration of endodontic materials into the dentinal tubules during root canal treatment ${ }^{1}$. Crown discoloration is well known; however, crown and root discoloration induced by root canal sealers can be different due to the arrangement of dentinal tubules in different areas of the tooth.

Root discoloration is relevant if periodontal plastic procedures are needed, especially in cases of localized gingival recession or surgery, including bone resection for patients requiring aesthetic treatments. Additionally, it is also an important factor when direct or indirect veneers or full crowns are indicated. Thus, root discoloration may influence the periodontal and/or prosthetic treatment, as well as the overall aesthetic results.

Several studies have demonstrated crown discoloration induced by endodontic sealers containing zinc oxide, calcium hydroxide, and resin cements ${ }^{2-6}$. The materials used for root filling may also induce tooth discoloration, particularly if left in the pulp chamber and above the gingival margin. The discoloration is usually seen in the cervical third of the crown since the overlying, which is a translucent and colorless structure, is thinner in this area ${ }^{7,8}$.

This discoloration may be prevented by complete removal of sealer remnants, cutting the filling material below the cementoenamel junction, or through the use of sealers that do not cause tooth discoloration 6 .

However, root canal cements usually cause discoloration because of the presence of unreacted components or the corrosion of some components owing to moisture and/or chemical interaction with dentine ${ }^{7,9}$. The color stability of endodontic sealers may also be affected by their different chemical components. The components of endodontic sealers that are related to color change of the tooth are bismuth trioxide? silver ${ }^{10,11}$, and iron oxide ${ }^{12}$.

Many studies have investigated the discoloring potential of root canal cements, 2,-7,11,13-15. However, several methodological differences - especially in whether the smear layer is removed, and how color change is determined (by either vision or computer analysis of digital images) - result in difficulties when interpreting the data'.

Therefore, this study investigated crown and root discoloration promoted by different endodontic sealers after root canal filling using spectrophotometric analysis. The study hypotheses were that there would be significant discoloration differences regarding (i) the tooth area evaluated (middle and cervical regions of the crown, and coronal third of the root); (ii) the endodontic sealer evaluated; and (iii) the time period after root canal filling.

\section{Material and Methods}

Eighty bovine incisors were extracted, cleaned using ultrasound to remove debris and extrinsic stains, and then stored in in water at room temperature.

The specimens were randomly divided into four groups $(n=20)$ according to the endodontic sealer: Endofill (Dentsply, Petrópolis, Brazil), Sealer 26 (Dentsply, Petrópolis, 
Table 1 - Description of the endodontic sealers used in the study.

\begin{tabular}{lc}
\hline Material (Manufacturer) & Composition \\
\hline Endofill (Dentsply, Petrópolis, Brazil) & $\begin{array}{c}\text { Powder: zinc oxide, hydrogenated resin, bismuth subcarbonate, } \\
\text { barium sulfate, sodium boroato. } \\
\text { Liquid: eugenol and sweet almond oil }\end{array}$ \\
\hline Sealer 26 (Dentsply, Petrópolis, Brazil) & $\begin{array}{c}\text { Powder: bismuth trioxide, calcium hydroxide, hexamethylene } \\
\text { tetramine, titanium dioxide. } \\
\text { Resin: epoxy bisphenol }\end{array}$ \\
\hline $\begin{array}{l}\text { AH Plus (Dentsply DeTrey, Konstanz, } \\
\text { Germany) }\end{array}$ & $\begin{array}{c}\text { Epoxy resin, zirconium oxide, iron oxide, calcium tungstate, and } \\
\text { silicone oil }\end{array}$ \\
\hline MTA Fillapex (Angelus, Londrina, Brazil) & $\begin{array}{c}\text { Paste A: salicylate resin, bismuth Trioxide, fumed silica. } \\
\text { Paste B: fumed Silica, titanium Dioxide, mineral. }\end{array}$ \\
\hline
\end{tabular}

Brazil), AH Plus (Dentsply DeTrey, Konstanz, Germany), and MTA Fillapex (Angelus, Londrina, Brazil). Their compositions are shown in Table 1.

Commission International de l'Eclairage $(\mathrm{CIE}) \mathrm{L} \mathrm{a}^{*} \mathrm{~b}$ parameters ${ }^{16}$ were recorded using Easyshade Spectrophotometer (VITA Zahnfabrik, Bad Säckingen, Germany) before preparation and root canal filling (baseline, T0). Color readings were always recorded on three assessment points: (i) the middle third of the crown (CM); (ii) the cervical third of the crown (2 mm above the cementoenamel junction) (CC); and (iii) the coronal third of the root, immediately below the cementoenamel junction (R). Each point was measured three times, and the final value was the mean of these readings.

Coronal opening was performed, followed by root canal treatment. The root canals were prepared using a crown-down technique and K-files; each canal was prepared to a size $90 \mathrm{~K}$-file (Denstply Maillefer, Ballaigues, Switzerland), which was the master apical file using 2.5\% sodium hypochlorite solution (Biodinâmica, Ibiporã, Brazil) and $17 \%$ ethylenediaminetetraacetic acid (EDTA) (Biodinâmica, Ibiporã, Brazil) as irrigant. The specimens were filled by Tagger's hybrid technique, using standardized (size 90) and nonstandardized (fine-medium size) gutta-percha cones (Denstply Maillefer, Ballaigues, Switzerland) as master and accessory cones (Denstply Maillefer, Ballaigues, Switzerland), respectively. Sections were then done $2 \mathrm{~mm}$ below the cementoenamel junction.

After cleaning, the pulp chamber was dried with cotton pellets, and the crown was sealed with glass ionomer cement (Shade A3, Vidrion R, S.S. White, Rio de Janeiro, Brazil).

Subsequent color readings were obtained at the following time periods after completion of endodontic treatment: 24 hours (T1), seven days (T2), 30 days (T3), and 90 days (T4). For standardization purposes, the same operator carried out all measurements.

The color difference $(\Delta \mathrm{E})$ between baseline and subsequent readings were calculated according to the equation:

$\Delta \mathrm{E}=\left[\left(\Delta \mathrm{L}^{*}\right)^{2}+\left(\Delta \mathrm{a}^{*}\right)^{2}+\left(\Delta \mathrm{b}^{*}\right)^{2}\right]^{1 / 2}$

where $\Delta L^{*}, \Delta a^{*}$ and $\Delta b^{*}$ are the differences between the parameters before (baseline) and after each period of time ( $\Delta \mathrm{E} 1=24$ hours and baseline; $\Delta \mathrm{E} 2=7$ days and baseline, $\Delta \mathrm{E} 3=30$ days and baseline and $\Delta \mathrm{E} 4=90$ days and baseline). When $\Delta \mathrm{E}$ was between 1 and 3.7, the color change was considered acceptable. Values higher than 3.7 were 
Table 2 - Means and standard deviations for $\Delta$ E values according to endodontic sealer, tooth region and time.

\begin{tabular}{|c|c|c|c|c|c|}
\hline Periods & Regions & Endofill & Sealer 26 & AH Plus & MTA Fillapex \\
\hline & $\mathrm{CM}$ & $3.59 \pm 1.86^{\mathrm{a}, \mathrm{b}, \mathrm{c}}$ & $4.15 \pm 1.34^{\mathrm{a}, \mathrm{b}, \mathrm{cod}}$ & $6.59 \pm 2.90^{\text {codeffg }}$ & $3.74 \pm 1.21^{a, b, c}$ \\
\hline \multirow[t]{3}{*}{ T1 } & $\mathrm{CC}$ & $4.11 \pm 1.19^{\mathrm{a}, \mathrm{b}, \mathrm{cod}}$ & $3.76 \pm 1.44^{\mathrm{a}, \mathrm{b}, \mathrm{c}}$ & $6.70 \pm 2.23^{\text {code.fog }}$ & $3.47 \pm 1.77^{a, b, o}$ \\
\hline & $\mathrm{R}$ & $13.23 \pm 5.47^{j k}$ & $4.64 \pm 2.46^{a, b, c, d}$ & $8.95 \pm 2.88^{f, g h, i}$ & $7.65 \pm 1.93^{\text {def.g., }}$ \\
\hline & $\mathrm{CM}$ & $2.70 \pm 1.87^{a, b}$ & $3.50 \pm 1.91^{a, b, c}$ & $6.84 \pm 4.64^{\text {code.fg }}$ & $3.47 \pm 1.50^{a, b, c}$ \\
\hline \multirow[t]{3}{*}{ T2 } & $\mathrm{CC}$ & $3.05 \pm 1.15^{\mathrm{a}, \mathrm{b}, \mathrm{o}}$ & $3.99 \pm 1.47^{\mathrm{a}, \mathrm{b}, \mathrm{cod}}$ & $5.44 \pm 2.04^{\text {a,bo,de, } f}$ & $3.04 \pm 1.59^{\mathrm{a}, \mathrm{b}, \mathrm{c}}$ \\
\hline & $\mathrm{R}$ & $16.66 \pm 7.50^{\mathrm{lm}}$ & $4.85 \pm 2.66^{\text {abodode }}$ & $8.67 \pm 3.51^{\text {efighi }}$ & $10.96 \pm 2.74^{\text {hi.k }}$ \\
\hline & $\mathrm{CM}$ & $2.22 \pm 1.27^{\mathrm{a}}$ & $4.11 \pm 1.73^{\mathrm{a}, \mathrm{b}, \mathrm{cod}}$ & $4.20 \pm 3.14^{a, b, o, d}$ & $3.34 \pm 1.46^{\mathrm{ab}, \mathrm{o}}$ \\
\hline \multirow[t]{3}{*}{ T3 } & $\mathrm{CC}$ & $2.38 \pm 1.34^{\mathrm{a} . \mathrm{b}}$ & $4.19 \pm 1.61^{\mathrm{a}, \mathrm{b}, \mathrm{cod}}$ & $5.07 \pm 3.31^{\text {a,bo,def } f}$ & $3.88 \pm 2.31^{\mathrm{ab}, \mathrm{a}, \mathrm{d}}$ \\
\hline & $\mathrm{R}$ & $13.96 \pm 4.91^{\mathrm{kl}}$ & $5.02 \pm 3.04^{\text {a b,ode }}$ & $8.58 \pm 3.31^{\text {ef,ghi, }}$ & $13.68 \pm 2.68^{\mathrm{j} k \mathrm{k}}$ \\
\hline & $\mathrm{CM}$ & $2.21 \pm 1.14^{\mathrm{a}}$ & $5.93 \pm 3.04$ abo,d,e, $f$ & $6.14 \pm 4.54$ bocdef & $4.47 \pm 1.74 \mathrm{abba,d}$ \\
\hline \multirow[t]{2}{*}{ T4 } & $\mathrm{CC}$ & $2.46 \pm 0.99^{a, b}$ & $6.59 \pm 3.76^{\text {cod,ef.,g }}$ & $5.30 \pm 2.26^{\text {abo, }, \text { def } f}$ & $5.64 \pm 1.85^{\text {a,bo, ade, } f}$ \\
\hline & $\mathrm{R}$ & $10.33 \pm 3.91$ ghij & $13.07 \pm 8.99 \mathrm{jkl}$ & $11.26 \pm 3.73^{\text {hijk }}$ & $19.20 \pm 2.30^{\mathrm{m}}$ \\
\hline
\end{tabular}

$\mathrm{CM}$, center of tooth crown; $\mathrm{CC}$, crown at $2 \mathrm{~mm}$ from the cementoenamel junction; $\mathrm{R}$, root right below the cementoenamel junction.

Note: Different letters indicated statistically significant differences between the tested groups. Three-way ANOVA and Tukey's HSD tests, $\mathrm{p}<0.05$.

considered clinically relevant and highly noticeable as considered in previously literature, with the same cut-off values using spectrophotometric analysis ${ }^{13,17}$.

Data were analyzed using three-way analysis of variance (ANOVA) (endodontic sealer, tooth region, and time) over repeated measurements and Tukey's honest significant difference (HSD) test with a significance level of 0.05 .

\section{Results}

The mean values of $\Delta \mathrm{E}$ with their standard deviations are presented in Table 2. Statistical analysis showed significant differences for all individual factors and the triple interactions $(p<0.01)$. All double interactions were statistically similar $(p>0.05)$.

In terms of individual endodontic sealers, sealer $26(\Delta E=5.32 \pm 4.21)$ exhibited the lowest $\Delta \mathrm{E}$ values. Endofill $(\Delta \mathrm{E}=6.41 \pm 6.21)$, MTA Fillapex $(\Delta \mathrm{E}=6.88 \pm 5.31)$, and $\mathrm{AH}$ Plus $(\Delta \mathrm{E}=6.98 \pm 3.77)$ exhibited higher $\Delta \mathrm{E}$ values, which were statistically similar.

In terms of tooth region, the largest color change was observed at the cervical third of the root $(\Delta E=10.67 \pm 5.87)$. Color changes at the middle third $(\Delta E=4.20 \pm 2.76)$ and cervical third of the crown $(\Delta E=4.32 \pm 2.38)$ were statistically similar. As for time, the largest $\Delta \mathrm{E}$ values were observed at $\mathrm{T} 4(\Delta \mathrm{E}=7.72 \pm 6.00)$. Measurements taken at $\mathrm{T} 1$ $(\Delta \mathrm{E}=5.88 \pm 3.73), \mathrm{T} 2(\Delta \mathrm{E}=6.10 \pm 5.08)$, and $\mathrm{T} 3(\Delta \mathrm{E}=5.89 \pm 4.72)$ were similar.

\section{Discussion}

The hypothesis regarding the difference in tooth discoloration between tooth areas was confirmed in this study, since the largest color change was observed at the cervical third of the root. Color changes observed at the middle and cervical thirds of the crown were statistically similar. 
Color changes at crown are the most evident and relevant in clinical practice, considering that this dental portion is always visible. However, in case of gingival plastic surgeries are necessary in anterior teeth, the most coronal portion of the root becomes as clinically and esthetically important as the dental crown.

The assessment point located at the cervical third of the root was associated with the highest $\Delta \mathrm{E}$ values. In this case, discoloration occurred due to the presence of filling material, gutta-percha, and endodontic sealer. Most studies that have evaluated the influence of endodontic sealers on tooth crown discoloration have employed only the endodontic sealer $2,4-7,11,13-15$. In the present study, the root canals were prepared and filled using gutta-percha and endodontic sealer, using a technique similar to that used in clinical situations. Gutta-percha is the most common core-filling material used worldwide, but it has been reported to cause a light pink discoloration 8,18 .

The cervical region of the crown exhibited the mildest discoloration, despite the greater quantity and diameter of dentinal tubules ${ }^{19}$. A possible explanation for this result is better cleaning of this region after root canal filling. Tooth discoloration also occurs due to the presence of remaining endodontic sealer inside the dentinal tubules. In the present study, sectioning of the root canal filling was standardized at $2 \mathrm{~mm}$ below the cementoenamel junction, and the pulp chamber was cleaned with cotton pellets and alcohol. This protocol differs from several studies that evaluated crown discoloration promoted by endodontic sealers in which only the pulp chamber or part of it was filled with endodontic sealer ${ }^{2-5}$.

Clinically, all sealer remnants should be removed from the pulp chamber during endodontic treatment; however, this does not always occur, and sealer remnants can result in color changes ${ }^{7}$. In the present study, a different protocol was used, in which the objective was to simulate clinical reality where the endodontic cement should not fill the pulp chamber and should be located below the cementoenamel junction.

The hypothesis that the endodontic sealer used for root canal filling may interfere with the color stability of the crown and root was confirmed in this study. Endodontic sealers usually cause color discoloration due to the presence of components that did not react or corrosion of some of these components in the presence of humidity and/or chemical interaction with dentin 7 . Some radiopacifiers such as bismuth oxide - present in some endodontic sealers, including Sealer 26 and MTA Fillapex - trigger a chemical interaction inside the root canal that leads to discoloration tending toward green or black ${ }^{1,9}$. There are reports in the literature that iron oxide, present in AH Plus, can cause grey discoloration of teeth ${ }^{12}$. Eugenol, present in Endofill, oxidizes and changes color over time, leading to staining of the dental structure?.

After analysis and comparison of $\Delta \mathrm{E}$ values, the group filled with Sealer 26 exhibited the mildest discoloration. This result differs from the findings of Meincke et al. ${ }^{4}$, in which the material exhibited the highest mean $\Delta \mathrm{E}$. This difference may be related to the longer evaluation period used here.

In the present study, AH Plus demonstrated a similar discoloration level as Endofill, corroborating the finding of Meincke et al. ${ }^{4}$. AH Plus is a resin cement containing zirconium oxide as a radiopacifier, a component that is not known to cause tooth discoloration ${ }^{9}$. However, the present study revealed relatively high $\Delta \mathrm{E}$ values when this sealer 
was used. This color change was also observed by Thomson et al. ${ }^{18}$, Meincke et al. ${ }^{4}$, and Forghani et al. ${ }^{6}$. Studies by Lenherr et al. ${ }^{3}$ and Forghani et al. ${ }^{6}$ revealed the greatest discoloration occurred during the first three months - which was the study period of the present investigation - and a reduction in $\Delta \mathrm{E}$ values after this period.

MTA Fillapex is an endodontic sealer recently introduced into the market that has characteristics similar to those of MTA 6,20 ; its potential for crown discoloration was previously analyzed5,6,21,22. The results of this study indicated that MTA Fillapex also had potential to induce color alterations, which was observed by Forghani et al. ${ }^{6}$, Gürel et al. ${ }^{21}$, and Suciu et al. ${ }^{22 ;}$; the results differed from those of Ioannidis et al. ${ }^{5}$, in which MTA Fillapex exhibited satisfactory color stability, not perceptible to the human eye.

Cements containing zinc oxide and eugenol, such as Endofill, have also been evaluated in several studies $2,4,8,11,13,22$; these studies have shown these cements promoted darkening of the tooth crown, probably due to the presence of eugenol. The chromogenic potential of zinc oxide and eugenol $(\mathrm{ZnOE})$ sealers has been attributed to the unstable chemical bond between zinc oxide and eugenol. Nevertheless, even after the end of the reaction, the oxidation of eugenol can cause tooth darkening with time ${ }^{7}$.

The hypothesis progressive tooth discoloration occurs over time was not confirmed in this study. Despite the difference in values, statistically these alterations not differed. This result is different than those observed by others ${ }^{4 \cdot 6,14,21}$. In the long-term, core materials and sealers interact with dentin. Any change to the optical and chromatic properties of the dentinal structure is likely to cause an alteration in the outward appearance of the crown due to its light transmitting and reflecting properties ${ }^{5,13}$.

After endodontic treatment, the tooth should maintain its function and its aesthetics; thus, it is important to select endodontic sealers that promote the least amount of tooth discoloration. This should be considered even in teeth with extensive coronal destruction that will receive prosthetic reconstructions for reestablishment of function, since masking of such alterations requires removal of a greater quantity of tooth structure, weakening the tooth and increasing the risk of tooth fracture in the future.

In sum, all endodontic sealers tested induced crown and root discoloration, and Sealer 26 caused the lowest amount of color change. Comparisons between tooth regions revealed that the smallest amount of color alteration was in the cervical third of the root. However, it is important consider that current study used bovine teeth instead of human teeth. Although all similarities, properties differences between human and bovine teeth must be considered when interpreting results obtained from any experiment with bovine teeth substrate ${ }^{23}$.

\section{Acknowledgements}

The authors deny any conflicts of interest related to this study.

\section{References}

1. Ahmed HMA, Abbott PV. Discolouration potential of endodontic procedures and materials: a review. Int Endod J. 2012 Oct;45(10):883-97. doi: 10.1111/j.1365-2591.2012.02071.x. 
2. Zare Jahromi M, Navabi AA, Ekhtiari M. Comparing coronal discoloration between $\mathrm{AH} 26$ and ZOE sealers. Iran Endod J. 2011 Fall;;6(4):146-9.

3. Lenherr P, Allgayer N, Weiger R, Filippi A, Attin T, Krastl G. Tooth discoloration induced by endodontic materials: a laboratory study. Int Endod J. 2012 Oct;45(10):942-9. doi: 10.1111/j.1365-2591.2012.02053.x.

4. Meincke DK, Prado M, Gomes BPF, Bona AD, Sousa ELR. Effect of endodontic sealers on tooth color. J Dent. 2013 Aug;41(Suppl 3):e93-6.

5. Ioannidis K, Mistakidis I, Beltes P, Karagiannis V. Spectrophotometric analysis of crown discoloration induced by MTA- and ZnOE-based sealers. J Appl Oral Sci. 2013 Mar-Apr;21(2):138-44. doi: 10.1590/1678-7757201302254.

6. Forghani M, Gharechahi M, Karimpour S. In vitro evaluation of tooth discolouration induced by mineral trioxide aggregate Fillapex and iRoot SP endodontic sealers. Aust Endod J. 2016 Dec;42(3):99-103. doi: 10.1111/aej.12144.

7. Parsons JR, Walton RE, Ricks-Williamson L. In vitro longitudinal assessment of coronal discoloration from endodontic sealers. J Endod. 2001 Nov;27(11):699-702.

8. Partovi M, Al-Havvaz AH, Soleimani B. In vitro computer analysis of crown discolouration from commonly used endodontic sealers. Aust Endod J. 2006 Dec;32(3):116-9.

9. Walsh LJ, Athanassiadis B. Endodontic aesthetic iatrodontics. Aust Dental Prac. 2007;18:62-4.

10. Allan NA, Walton RC, Schaeffer MA. Setting times for endodontic sealers under clinical usage and in vitro conditions. J Endod. 2001 Jun;27(6):421-3.

11. Davis MC, Walton RE, Rivera EM. Sealer distribution in coronal dentin. J Endod. 2002 Jun;28(6):464-6.

12. Asgary S, Parirokh M, Eghbal MJ, Brink F. Chemical differences between white and gray mineral trioxide aggregate. J Endod. 2005 Feb;31(2):101-3.

13. Ioannidis K, Beltes P, Lambrianidis T, Kapagiannidis D, Karagiannis V. Validation and spectrophotometric analysis of crown discoloration induced by root canal sealers. Clin Oral Investig. 2013 Jul;17(6):1525-33. doi: 10.1007/s00784-012-0850-x.

14. Kohli MR, Yamaguchi M, Setzer FC, Karabucak B. Spectrophotometric analysis of coronal tooth discoloration induced by various bioceramic cements and other endodontic materials. J Endod. 2015 Nov;41(11):1862-6. doi: 10.1016/j.joen.2015.07.003.

15. Shokouhinejad N, Nekoofar MH, Pirmoazen S, Shamshiri AR, Dummer PM. Evaluation and comparison of occurrence of tooth discoloration after the application of various calcium silicate-based cements: an ex vivo study. J Endod. 2016 Jan;42(1):140-4. doi: 10.1016/j.joen.2015.08.034.

16. International Commission on Illumination. Recommendations on uniform color spaces, colordifference equations, psychometric color terms. Bureau Central de la CIE. 1978;15:13.

17. Johnston WM, Kao EC. Assessment of appearance match by visual observation and clinical colorimetry. J Dent Res. 1989 May;68(5):819-22.

18. Thomson AD, Athanassiadis B, Kahler B, Walsh L. Tooth discolouration: staining effects of various sealers and medicaments. Aust Endod J. 2012 Apr;38(1):2-9. doi: 10.1111/j.1747-4477.2011.00339.x.

19. Costa BM, Iwamoto AS, Puppin-Rontani RM, Pascon FM. Comparative analysis of root dentin morphology and structure of human versus bovine primary teeth. Microsc Microanal. 2015 Jun;21(3):689-94. doi: 10.1017/S1431927615000434.

20. Lisbôa El, Silva Neto UX da, Carneiro E, Fariniuk LF, Westphalen VPD. Capacidade de selamento e penetração intratubular do MTA Fillapex e do AH Plus em dentes humanos. Rev Odontol UNESP. 2017;46(1):7-13. doi: 10.1590/1807-2577.25915. 
21. Gürel MA, Kivanç BH, Ekici A, Alaçam T. Evaluation of crown discoloration induced by endodontic sealers and change ratio determination after bleaching. Aust Endod J. 2016 Dec;42(3):119-123. doi: 10.1111/aej.12147.

22. Suciu I, Ionescu E, Dimitriu BA, Bartok RI, Moldoveanu GF, Gheorghiu IM, et al. An optical investigation of dentinal discoloration due to commonly endodontic sealers, using the transmitted light polarizing microscopy and spectrophotometry. Rom J Morphol Embryol. 2016;57(1):153-9.

23. Yassen GH, Platt JA, Hara AT. Bovine teeth as substitute for human teeth in dental research: a review of literature. J Oral Sci. 2011 Sep;53(3):273-82. 